\section{Diagnosis and \\ management of \\ hyperadrenocorticism \\ in dogs attending UK \\ primary-care practice}

\section{Imogen Schofield ${ }^{1}$, David Brodbelt ${ }^{1}$, Stijn Niessen ${ }^{1}$, Anna Wilson ${ }^{2}$, Dan O'Neill ${ }^{1}$}

1 Royal Veterinary College, London, United Kingdom

2 University of Cambridge, Cambridge, United Kingdom

\section{OBJECTIVES}

To describe the diagnosis and management of hyperadrenocorticism in dogs attending primary-care practice in the UK.

\section{METHODS}

The VetCompass database contains electronic patient records for dogs attending UK primary-care practices. The records of dogs diagnosed with hyperadrenocorticism were reviewed and additional diagnostic and management data were extracted including: diagnostic testing, treatment, trilostane dose and monitoring cortisol measurements.

\section{RESULTS}

The study identified 219 dogs diagnosed with hyperadrenocorticism. The most commonly used diagnostic tests were the ACTH stimulation test ( $n=206$ cases, $94.1 \%)$, low-dose dexamethasone suppression test $(70,32.0 \%)$ and urine cortisol-creatinine ratio (58, $26.5 \%)$. Differentiation between pituitary- and adrenaldependent aetiology was performed in 44 cases (20.1\%). Trilostane was given in 206 cases (94.1\%) with a mean starting dose of $3.30 \mathrm{mg} / \mathrm{kg} /$ day (SD 1.28). Ninety-four cases $(45.6 \%)$ had no changes made to their initial trilostane dose. Of dogs with cortisol concentrations recorded after initiation of therapy, post-ACTH stimulation cortisol did not drop below the upper reference range of $250 \mathrm{nmol} / \mathrm{L}$ in any test in $32(32.0 \%)$ and results fell below the lower reference range of $40 \mathrm{nmol} / \mathrm{L}$ in 21 (21.0\%). latrogenic hypoadrenocorticism or a suspected adverse response was recorded in 28 (13.6\%) dogs receiving trilostane.

\section{STATEMENT (CONCLUSIONS)}

This study provides benchmark data describing the diagnosis and management of hyperadrenocorticism in primary-care practice. Findings show the ACTH stimulation test predominates in diagnosis and differentiation between pituitary and adrenal disease is infrequently performed, contrary to current expert recommendations. Further work evaluating the benefits of disease differentiation and survival after treatment in a primary-care setting is merited.
The Cushing's clinical score: development of a primary-care practice tool to quantify the clinical signs of dogs with hyperadrenocorticism

\section{Imogen Schofield, Dan O'Neill, David Brodbelt, Stijn Niessen}

Royal Veterinary College, London, United Kingdom

\section{OBJECTIVES}

With doubts raised over the accuracy of hormonal testing of hyperadrenocorticism, monitoring recommendations for dogs undergoing trilostane treatment emphasise the importance of clinical sign assessment. This study aimed to produce a tool to enable standardised quantification of the clinical signs associated with hyperadrenocorticism in primary-care practice.

\section{METHODS}

Structured focus groups were held with 19 clinicians and 13 owners of dogs with hyperadrenocorticism, 21 veterinary publications were reviewed and 20 randomly selected electronic health records of hyperadrenocorticism cases were analysed to identify potential clinical signs associated with hyperadrenocorticism. Qualitative thematic analysis identified five categories of clinical signs. A scoring system was designed based on the severity of clinical signs in each category ranging from $0-3$, producing a score out of 15 (higher score indicate more clinical hyperadrenocorticism). The tool was piloted with 16 owners and 12 clinicians to assess its readability and clarity.

\section{RESULTS}

A list of 24 clinical signs associated with hyperadrenocorticism was drafted. Qualitative thematic analysis identified polydipsia and polyphagia as the most commonly 


\section{Oral presentations}

discussed clinical signs in the focus groups, with changes in demeanor and lethargy more frequently mentioned by owners than clinicians. Clinical signs were summarised into five categories: drinking, urination, appetite, appearance and attitude/activity. Clinicians and owners reported the tool to be clear, concise and practical for a primarycare setting.

\section{STATEMENT (CONCLUSIONS)}

The developed tool standardises the recording of clinical signs of hyperadrenocorticism and could be used in practice to assess medical management of hyperadrenocorticism. Further validation is planned through correlation with biological parameters and patient outcomes.

\section{Breed influence}

on canine thyroid reference intervals and age-related thyroid decline

\section{Olivia Barnard-Jones ${ }^{1}$, Kent Refsal ${ }^{2}$, Peter Graham ${ }^{1}$}

1 University of Nottingham, Sutton Bonington, United Kingdom

2 Michigan State University, East Lansing, USA

\section{OBJECTIVES}

Hypothyroidism is a common canine condition which can be difficult to diagnose. Misdiagnosis can result in unnecessary life-long treatment. One complicating factor may be variation in thyroid hormone reference intervals between breeds, previously shown to be diagnostically important in sighthounds. Increasing age is associated with decreasing thyroxine and increasing TSH serum concentrations, but breed influence on this effect has not previously been explored.

This projects aimed to investigate whether more, non-sighthound, breed-specific reference intervals would be appropriate and to discover whether breed influenced age-related thyroid changes.

\section{METHODS}

The study explored total and free, thyroxine and 3-5-3'-triiodothyronine (TT4, FT4, TT3, FT3), and thyrotropin (TSH) in 81,985 euthyroid dogs from 214 breeds using a database of serum thyroid profile results. Breed reference intervals (2.5 to 97.5 percentile) were created for 77 breeds with more than 140 cases.

\section{RESULTS}

Breed-specific reference limits differed; the lower limit for TT4 ranged from 7 (Sharpei) to $17 \mathrm{nmol} / \mathrm{L}$ (Cavalier King Charles Spaniel; CKCS) and the upper TSH limit from 0.44 (Soft Coated Wheaten Terrier) to $0.63 \mathrm{ng} / \mathrm{ml}$ (Keeshond). There was a breed effect on age-related thyroid hormone decline and TSH increase. TT4 decline ranged from -0.021 (Basenji) to -1.324 (CKCS) nmol/L/yr. TSH increase ranged from 0.00097 (Bulmastiff) to 0.0137 (CKCS) $\mathrm{ng} / \mathrm{ml} / \mathrm{yr}$.

\section{STATEMENT (CONCLUSIONS)}

Breeds other than the sighthound group would benefit from their own breed-specific thyroid reference intervals, such as CKCS, as they differed significantly from all-breed intervals with a consequent risk of misdiagnosis. Some breeds also have quicker "thyroid-axis-ageing" than others.

\section{Factors influencing thyroid function and deiodinase indices}

\section{Emma Campbell ${ }^{1}$, Kent Refsal ${ }^{2}$, Peter Graham ${ }^{1}$}

1 University of Nottingham, Sutton Bonington, United Kingdom 2 Michigan State University, East Lansing, USA

\section{OBJECTIVES}

Age, breed, gender and obesity have been shown to influence thyroid function and concentrations of thyroid hormones in the dog. This study investigated whether these factors, breed size and geographical location (climate) influenced deiodinase activity (tri-iodothyronine (T3): thyroxine (T4) ratio) and thyroid functional 'setpoint' (T4:Thyrotropin (TSH) ratio).

\section{METHODS}

Analysis of a USA database of canine laboratory thyroid function assessments ( $\mathrm{n}$ 70,000 euthyroid cases) Regression was used to assess associations between numerical values and Kruskal-Wallis to compare differences between categories.

\section{RESULTS}

Age was associated with an increased deiodinase activity $(P<0.05)$ and lower thyroid functional 'set-point' $(P<0.001)$. 'Set-point' was affected by breed $(P<0.001)$ and T4:TSH ratio was lower in overweight dogs $(P<0.05)$ Overweight was associated with higher T3, T4 and TSH concentrations $(p<0.001)$ but there was no difference in 